\title{
OPTIMAL DATA PLACEMENT IN TWO-HEADED DISK SYSTEMS
}

\author{
YANNIS MANOLOPOULOS and J. (YANNIS) G. KOLLIAS*
}

Divison of Computer and Electronics Engineering, Department of Electrical Engineering, Aristotelian University of Thessaloniki, 54006 Thessaloniki, Greece.

\author{
Division of Computer Science, \\ Department of Electrical Engineering, \\ National Technical University of Athens, \\ 15773 Athens, Greece
}

\begin{abstract}
.
A problem inherent to the performance of disk systems is the data placement in cyinders in such a way that the seek time is minimized. If successive searchers are independent, then the optimal placement for conventional one-headed disk systems is the organ-pipe arrangement. According to this arrangement the most frequent cylinder is placed in the central location, while the less frequent cylinders are placed right and left alternatively. This paper proves that the optimal placement for two-headed disk systems is the "camel" arrangement, which may be viewed as two consecutive organ-pipe arrangements. It is also proved that, for a two-headed disk system with $N=2(2 n+1)$ cylinders, the total number of these optimal camel arrangements is $\exp _{2}(N / 2+1)$.
\end{abstract}

CR categories and subject descriptors: D.4.2.

General terms : Algorithms, Performance.

Additional key-words and phrases: Camel arrangement, Data placement, Organ-pipe arrangement, Seek distance, Two-headed disk.

\section{Introduction.}

The organ-pipe arrangement has been proved to be optimal with respect to the minimizaton of the expected distance traveled by the moving arm of a single-headed disk system. According to this technique the cylinders are ordered in descending sequences of their access probabilities. The most frequent cylinder is placed in the central position of the storage medium and then even (odd) numbered cylinders are placed alternatively to the right (left). Symmetrically, even (odd) numbered cylinders may be positioned to the left (right) of the center. It has been proved also that for every set of cylinders there are two optimal data arrangements. Grossman and Silverman [3], Hardy, Littlewood and Polya [4] and Wong [6] provide three different proofs on the optimally of the organ-pipe arrangement.

* Professor J. G. Kollias died untimely December 1989.

Received May 1989. Revised November 1989. 
Two-headed disk systems are characterized by a fixed head separation distance which is measured by the number of cylinders between the two heads. The problem examined in this study is the optimal cylinder arrangement in two-headed disk systems, given their access probabillities. In the next section it is proved that the optimal data placement for these systems is the "camel" arrangement, which may be viewed as two consecutive organ-pipe arrangements. It is proved, also, that the number of optimal camel arrangements in a two-headed disk system with $N=2(2 n+1)$ cylinders is $2^{2 n+2}$, where $n$ is a positive integer. The last section draws some conclusions and discusses possible extensions.

\section{The camel arrangement.}

It is evident that the organ-pipe arrangement is not optimal in two-headed disk systems. Suppose that $N$ cylinders have to be arranged, where without loss of generality $N=2(2 n+1)$. In [5] it has been proved that if the disk is operating under the SCAN scheduling policy then the optimum head separation distance of the $\mathrm{read} / \mathrm{write}$ mechanism is $N / 2-1=2 n$. Each cylinder has been assigned an access probability $p_{i}$, where $\Sigma p_{i}=1$. The event of visiting a cylinder with a specific probability $p_{r}$ is independent of the previous cylinder visit with access probability $p_{s}$. Therefore, $p_{r} p_{s}$ is the probability that cylinders $r$ and $s$ may be visited one after the other. The distance covered by the moving mechanism is $d(r-s)$. Since the heads of the mechanism lie $2 n$ positions away from each other, the function $d(r-s)$ is defined as follows:

$$
d(r-s)=\begin{array}{ll}
|r-s| & \text { if } 1 \leq r, s \leq N / 2 \text { or } N / 2+1 \leq r, s \leq N \\
|r-s-N / 2| & \text { if } N / 2+1 \leq r \leq N \text { and } 1 \leq s \leq N / 2 \\
|r-s+N / 2| & \text { if } 1 \leq r \leq N / 2 \text { and } N / 2+1 \leq s \leq N
\end{array}
$$

Thus the expected cost for traveling from cylinder $r$ to cylinder $s$ is $p_{r} p_{s} d(r-s)$. If all the possible position pairs are taken into consideration, the total expected cost is:

$$
E=\sum_{r=1}^{n} \sum_{s=1}^{n} p_{r} p_{s} d(r-s)=P^{T} C P
$$

where the element $P(i)$ of the probability vector $P$ gives the probability of visiting position $i, C$ is the cost matrix with elements $C(i, j)$ giving the cost for moving from position $i$ to position $j$. Due to physical disk characteristics the matrix $C$ may be divided into four equal submatrices $C^{\prime}$ with $N / 2 \times N / 2$ elements each:

$$
C=\left[\begin{array}{ll}
C^{\prime} & C^{\prime} \\
C^{\prime} & C^{\prime}
\end{array}\right]
$$

The vector $P$ may similarly be defined as:

$$
P=\left[\begin{array}{l}
P^{\prime} \\
P^{\prime \prime}
\end{array}\right]
$$


Combining relations (2), (3) and (4) we find

$$
E=P^{T} C P=Q^{T} C^{\prime} Q
$$

where:

$$
Q=P^{\prime}+P^{\prime \prime}
$$

Assume that the probabilities $p_{i}$ are not equal to each other. Without loss of generality, let:

$$
p_{1}>p_{2}>\ldots p_{n}>0 \text {. }
$$

Suppose also that vector $P$ is divided in the two subvectors $P^{\prime}$ and $P^{\prime \prime}$ in such a way that:

$$
P^{\prime}(i)=p_{2 i-1} \text { and } P^{\prime \prime}(i)=p_{2 i} \text {, where } 1 \leq i \leq N / 2 \text {. }
$$

From relations (7) and (8) follows that the elements of the vectors $P^{\prime}$ and $P^{\prime \prime}$ with the same index $i$ designate a compound position.

$$
P^{\prime}=\left[\begin{array}{l}
p_{1} \\
p_{3} \\
\vdots \\
p_{n-1}
\end{array}\right] \text { and } P^{\prime \prime}=\left[\begin{array}{l}
p_{2} \\
p_{4} \\
\vdots \\
P_{n}
\end{array}\right]
$$

It is evident that:

$$
\begin{aligned}
& P^{\prime}(1)>P^{\prime}(2)>\ldots>P^{\prime}(N / 2) \quad \text { and } \\
& P^{\prime \prime}(1)>P^{\prime \prime}(2)>\ldots>P^{\prime \prime}(N / 2) .
\end{aligned}
$$

Summing these relations we get

$$
Q(1)>Q(2)>\ldots>Q(N / 2) \text {. }
$$

The problem of minimizing expression (2) has been transformed to minimization of expression (5). The elements of the vector $Q$ have to be ordered according to the organ pipe arrangement. Since the elements of $P^{\prime}$ and $P^{\prime \prime}$ with the same index have to occupy compound positions, it is certain that the elements of both $P^{\prime}$ and $P^{\prime \prime}$ have to be arranged as organ pipe permutations. In this way the elements of $P^{\prime}$ and $P^{\prime \prime}$, ordered in a linear row, form a camel-like arrangement.

Next, it is proved that the number of optimal camel arrangements is $2^{N / 2+1}$. The problem may be stated: "what is the number of ways to store the elements of the vectors $P^{\prime}$ and $P^{\prime \prime}$ in $N / 2$ compound storage locations?". If the rule followed is: "every even (odd) pair of cylinders is stored by moving to the left (right) of the central compound pair", then as a direct consequence, every single storage position may take either of two cylinders. Therefore, the possible number of ways that the $N$ cylinders may be stored in the $N$ positions is $2^{N / 2}$. If the equivalent rule: "every even (odd) pair of cylinders is stored by moving to the right (left) of the central compound pair" is accepted, then another $2^{N / 2}$ optimal placements are produced. Finally, the result follows by summation. 


\section{Conclusions.}

We have proved that for two-headed disk systems there are $2^{(N / 2+1)}$ optimal cylinder arrangements, with respect to minimization of the seek time of the moving mechanism. These arrangements are called camel arrangements. It may be generalized that in disk systems with $k$ read/write heads per surface, the number of optimal data placements is $2(k !)^{N / k}$ and may be viewed as $k$ successive organ-pipe arrangements.

Distribution $Q$ (of section 3) is produced from distributions $P^{\prime}$ and $P^{\prime \prime}$ by adding elements with the same index. Many distributions could be produced in place of $Q$ by combining one element from each of the two initial distributions but in a different way. It should be kept in mind also, that the elements of the $P^{\prime}$ and $P^{\prime \prime}$ distributions are arranged in descending value. Therefore, $Q$ is the distribution with the greatest entropy since it differs most from the uniform distribution when compared to all the other ones that may be produced from $P^{\prime}$ and $P^{\prime \prime}$. This remark agrees with the theoretical result of [2] that the assumption of "uniformity of queries" leads to pessimistic cost estimates.

A possible extension could deal with the dynamic version of this problem, e.g. the access probabilities are not known in advance or change with time. In addition, future research could examine how sensitive the optimal solution is to the variation in separation distance and calculate the error inserted in seek estimations due to the camel arrangement under the assumption that cylinder visits are probabilistically dependent [1]. Future research could, also, provide algorithms for recognizing if a cylinder permutation is already optimal in the camel arrangement sense.

\section{Acknowledgement.}

We wish to thank Mr. Nikos Evagelopoulos for his valuable assistance in the preparation of this work and one of the anonymous referees for his useful comments.

\section{REFERENCES}

[1] Carson S. D. and Vongsathorn P.: Error bounds on disk arrangements using frequency information, Information Processing Letters, Vol. 31, pp. 209-213, 1989.

[2] Christodoulakis S.: Implications of certain assumptions in database performance evaluation, Transactions on Database Systems of the ACM, Vol. 9, No. 2, pp. 163-186, 1984.

[3] Grossman D. D. and Silverman F. H.: Placement of records on a secondary storage device to minimize access time, Journal of the ACM, Vol. 20, No. 3, pp. 429-438, 1973.

[4] Hardy G. H., Littlewood J. E. and Polya G.: Inequalities, Cambridge University Press, Cambridge, England, 1952.

[5] Manolopoulos Y. and Kollias J. G.: Performance of a two-headed disk system when serving database queries under the SCAN policy, ACM Transactions on Database systems, Vol. 14, No. 3, 1989.

[6] Wong C. K.: Algorithmic Studies in Mass Storage Systems, Computer Science Press, 1983. 